\title{
Additive Results on Generalized Drazin Inverse in Minkowski Space $M$
}

\author{
D. Krishnaswamy and S. Anusuya \\ Department of Mathematics, Annamalai University, Annamalainagar-608 002, Tamilnadu, \\ India.
}

Abstract: In this paper we study the additive properties of generalized Drazin inverse of two Drazin invertible operators in Minkowski spaceM in terms of $P Q=Q P:$ Further we have derived the explicit representations of generalized Drazin inverse $(P+Q) y G$ interms of $P ; Q ; P y G$ and $Q y G$ in Minkowski space $M$.

Keywords: Generalized Drazin Inverse, Minkowski Inverse. AMS subject classification 15A09.

\section{Introduction}

Throughout we shall deal with $C^{n \times n}$, the space $n \times n$ complex matrices. Let $C^{n}$ be the space of complex $n$-tuples, we shall index the components of a complex vector in $C^{n}$ from 0 to $n-1$, that is $u=\left(u_{0}, u_{1}, u_{2}, \cdots, u_{n-1}\right)$. Let $G$ be the Minkowski metric tensor defined by $G_{u}=\left(u_{0},-u_{1},-u_{2}, \cdots,-u_{n-1}\right)$. Clearly the Minkowski metric matrix

$$
G=\left[\begin{array}{cc}
1 & 0 \\
0 & -1_{n-1}
\end{array}\right], G=G^{*} \text { and } G^{2}=I_{n}
$$

In [13], Minkowski inner product on $C^{n}$ is defined by $(u, v)=\left[u, G_{v}\right]$, where $[\cdot, \cdot]$ denotes the conventional Hilbert (unitary) space inner product. A space with Minkowski inner product is called a Minkowski space and denoted as $\mathscr{M}$.

For $A \in C^{n \times n}, x, y \in C^{n}$, by using (1.1)

$$
\begin{aligned}
(A x, y) & =[A x, G y] \\
& =\left[x, A^{*} G y\right] \\
& =\left[x, G\left(G A^{*} G\right) y\right] \\
& =[x, G \tilde{A} y], \text { where } \tilde{A}=G A^{*} G \\
& =(x, \tilde{A} y)
\end{aligned}
$$

The matrix $\tilde{A}$ is called the Minkowski adjoint of $A$ in $\mathscr{M}\left(A^{*}\right.$ is usual Hermitian adjoint of $A$ ). Naturally we call a matrix $A \in C^{n \times n} m$-symmetric in $\mathscr{M}$ if $A=\tilde{A}$. From the definition $\tilde{A}=G A^{*} G$ we have the following equivalence: $A$ is $m$-symmetric $\Leftrightarrow A G$ is hermitian $\Leftrightarrow G A$ is hermitian.

For $A \in C^{n \times n}, r k(A), N(A)$ and $R(A)$ are respectively the rank of $A$, null space of $A$ and range space of $A$. By a generalized inverse of $A$ we mean a solution of the equation $A X A=A$ and is denoted as $A^{(1)} \cdot A\{1\}$ is the set of all generalized inverses of $A$. Throughout $I$ refers to identity matrix of appropriate order unless otherwise specified. 
Definition $1.1[1]$

For $A \in C^{m \times n}, A^{\dagger}$ is the Moore-Penrose inverse of $A$ if $A A^{\dagger} A=A, A^{\dagger} A A^{\dagger}=$ $A^{\dagger}, A A^{\dagger}$ and $A^{\dagger} A$ are Hermitian. The Minkowski inverse of $A$, analogous to Moore-Penrose inverse of $A$ is introduced and its existence is discussed in [12].

Definition $1.2[12]$

For $A \in C^{m \times n}, A^{m}$ is the Minkowski inverse of $A$ if $A A^{m} A=A, A^{m} A A^{m}=$ $A^{m}, A A^{m}$ and $A^{m} A$ are $m$-symmetric.

\section{Lemma 1.3}

For $A \in C^{n \times n}$ then $A^{\dagger} G$ is the Moore-Penrose inverse of $G A$ in Minkowski's space $\mathscr{M}$, where $G$ is the Minkowski metric tensor of order $n$.

For $A \in B(X)$, if there exist an operator $A^{\dagger} G \in B(X)$ satisfying the following three operator equations [9].

$$
A A^{\dagger} G=A^{\dagger} G A, A^{\dagger} G A A^{\dagger} G=A^{\dagger} G, A^{k+1} A^{\dagger} G=A^{k} .
$$

Then $A^{\dagger}$ is called Drazin inverse of $A$. The smallest $k$ such that $r k\left(A^{k+1}\right)=$ $r k\left(A^{k}\right)$ holds is called index of $A$, denoted by $\operatorname{ind}(A)$. Notice also that $\operatorname{ind}(A)$ (if it finite) is the smallest non-negative integers $k$ such that $R\left(A^{k+1}\right)=R\left(A^{k}\right)$ and $N\left(A^{k+1}\right)=N\left(A^{k}\right)$.

The conditions (1.3) are equivalent to $A A^{\dagger} G=A^{\dagger} G A, A^{\dagger} G A A^{\dagger} G=A^{\dagger} G$, $A-A^{2} A^{\dagger} G$ is nilpotent.

The concept of generalized Drazin inverse on an infinite-dimensional Banach space was introduced by Koliha [11], which is the element $A^{m} \in B(x)$ such that

$$
A A^{m}=A^{m} A, A^{m} A A^{m}=A^{m}, A-A^{2} A^{m} \text { is quasi nilpotent. }
$$

If $A$ is generalized Drazin invertible, then the spectral idempotent $P$ of $A$ corresponding to $\{0\}$ is given by $P=I-A A^{m}$. The operator matrix form of $A$ with respect to the space decomposition $X=N(P) \oplus R(P)$ is given by $A=A_{1} \oplus A_{2}$, where $A_{1}$ is invertible and $A_{2}$ is quasi-nilpotent [1].

In recent years, the characterizations of the Drazin inverses of matrices or operators on an infinite-dimensional space have been considered by many authors (cf.[2-4]), Castro - Gonzalez et al, [2], Djordjevic and Wei [8], Hartwig et al, [10] have studied the generalized Drazin inverse on a Banach space. some additive properties and the explicit expression for the GD-inverse of the sum, are obtained in $[3,4,5,7]$. 
In this paper, using the technique of block operator matrices, we will investigate explicit representations of the generalized Drazin inverse $(P+Q)^{\dagger} G$ in term of $P, P^{\dagger} G, Q$ and $Q^{\dagger} G$ under the condition of $P Q=Q P$. Our results are improvement over the main results of [6]. Indeed, a totally new approach is provided to express the GD-inverse.

This paper is organized as follows. An explicit formula for $(P+Q)^{\dagger} G$ is presented in section 2. This is a key step of the paper. In section 3 , special cases are given to indicate the various applications of our main results.

\section{Main Result}

In the first part of this section, we give new expression for the GD-inverse of $P+Q$ in term of $P, P^{\dagger} G, Q$ and $Q^{\dagger} G$. It is interesting to note that our result is quite different from the expression for $(P+Q)^{\dagger} G$ in [8].

\section{Theorem 2.1}

Let $P, Q \in B(x)$ be GD-invertible in Minkowski space $\mathscr{M}$ and $P Q=Q P$. Then $P+Q$ is GD-invertible if and only if $I+P^{\dagger} G Q$ is GD-invertible in $\mathscr{M}$. In this case we have,

$$
\begin{aligned}
(P+Q)^{\dagger} G= & P^{\dagger}\left(I+P^{\dagger} Q\right)^{\dagger} G Q Q^{\dagger} G+\left(I-Q Q^{\dagger} G\right)\left[\sum_{n=0}^{\infty}(-Q)^{n}\left(P^{\dagger} G\right)^{n+1}\right] G+ \\
& {\left[\sum_{n=0}^{\infty}\left(Q^{\dagger} G\right)^{n+1}(-P)^{n}\right] G\left(I-P P^{\dagger} G\right) }
\end{aligned}
$$

and

$$
\begin{aligned}
(P+Q)(P+Q)^{\dagger} G= & \left(I+P^{\dagger} Q\right)^{\dagger} G\left(P P^{\dagger}+Q P^{\dagger}\right)\left[I+\left(Q Q^{\dagger} G\right)\right]+ \\
& \left(I+Q^{\dagger} P\right)^{\dagger}\left(P Q^{\dagger}+Q Q^{\dagger}\right)\left(I-P P^{\dagger} G\right) .
\end{aligned}
$$

\section{Proof:}

Since $P$ is GD-invertible, so we can write $P=P_{0} \oplus P_{00}$, where $P_{0}$ invertible, $P_{00}$ is Quasi - Nilpotent.

Also $P Q=Q P$, we can decompose $Q=Q_{0} \oplus Q_{00}$, where $Q_{0}$ and $Q_{00}$ are GDinvertible such that $P_{0} Q_{0}=Q_{0} P_{0}$ and $P_{00} Q_{00}=Q_{00} P_{00}$.

In a similar way, we can conclude that

$$
\begin{array}{ll}
P_{0}=P_{1} \oplus P_{2}, & P_{00}=P_{3} \oplus P_{4} \text { and } \\
Q_{0}=Q_{1} \oplus Q_{2}, & Q_{00}=Q_{3} \oplus Q_{4},
\end{array}
$$


where $P_{i}(i=1,2), Q_{j}(j=1,3)$ are invertible, $P_{m}(m=3,4), Q_{n}(n=2,4)$ are quasi-Nilpotent and $P_{i} Q_{i}=Q_{i} P_{i} \quad(i=1,2,3,4)$.

We have $(P+Q)=\left(P_{1}+Q_{1}\right) \oplus\left(P_{2}+Q_{2}\right) \oplus\left(P_{3}+Q_{3}\right) \oplus\left(P_{4}+Q_{4}\right)$.

Since $P_{2}$ is invertible, $Q_{2}$ is Quasi nilpotent and $P_{2} Q_{2}=Q_{2} P_{2}$,

we have $\rho\left(P_{2}+Q_{2}\right) \subset \rho\left(P_{2}^{\dagger}\right) \rho\left(Q_{2}\right)=\{0\}$.

Thus $P_{2}^{\dagger} Q_{2}$ is Quasi-Nilpotent and $I+P_{2}^{\dagger} G Q_{2}$ is invertible and

$0 \oplus\left(P_{2}+Q_{2}\right)^{\dagger} G \oplus 0 \oplus 0=0 \oplus P_{2}^{\dagger}\left(I+P_{2}^{\dagger} G Q_{2}\right)^{\dagger} G \oplus 0 \oplus 0$

$$
\begin{aligned}
& =0 \oplus P_{2}^{\dagger}\left(I+P_{2}^{\dagger} G Q_{2}\right)^{\dagger} G \oplus 0 \oplus 0 \\
& =0 \oplus P_{2}^{\dagger}\left[\sum_{n=0}^{\infty}\left(P_{2}^{\dagger}\right)^{n}\left(-Q_{2}\right)^{n}\right] G \oplus 0 \oplus 0 \\
& =\left(I-Q Q^{\dagger} G\right)\left[\sum_{n=0}^{\infty}\left(P^{\dagger}\right)^{n+1}(-Q)^{n}\right] G .
\end{aligned}
$$

Similarly we can prove that $P_{3} Q_{3}^{\dagger}$ is quasi-Nilpotent and $I+P_{3} Q_{3}^{\dagger}$ is invertible with

$\mathbf{0} \oplus \mathbf{0} \oplus\left(P_{3}+Q_{3}\right)^{\dagger} \mathbf{0}=\mathbf{0} \oplus \mathbf{0} \oplus Q_{3}^{\dagger}\left(I+Q_{3}^{\dagger} P_{3}\right)^{\dagger} G \oplus \mathbf{0}$

$$
\begin{aligned}
& =0 \oplus \mathbf{0} \oplus Q_{3}^{\dagger}\left[\sum_{n=0}^{\infty}\left(Q_{3}^{\dagger}\right)^{n}\left(-P_{3}\right)^{n}\right] \oplus 0 \\
& =\left[\sum_{n=0}^{\infty}\left(Q^{\dagger}\right)^{n+1}(-P)^{n}\right] G\left(I-P P^{\dagger} G\right) .
\end{aligned}
$$

Since $P_{4}$ and $Q_{4}$ are Quasi-Nilpotent and $P_{4} Q_{4}=Q_{4} P_{4}$,

we get that $P_{4}+Q_{4}$ is GD-invertible and $\left(P_{4}+Q_{4}\right)^{\dagger} G=0$.

Hence $P+Q$ is GD-invertible, if $P_{1}+Q_{1}$ is GD-invertible.

Note that $P_{1}, P_{1}^{\dagger} G, Q_{1}, Q_{1}^{\dagger} G, P_{1}+Q_{1}$ and $\left(P_{1}+Q_{1}\right)$ commute.

It is easy to know $\left(P_{1}+Q_{1}\right)^{\dagger} G$ is GD-Invertible, if $I+P^{\dagger} Q$ is GD-Invertible.

Thus $\left(P_{1}+Q_{1}\right)^{\dagger} G \oplus \mathbf{0} \oplus \mathbf{0} \oplus \mathbf{0}=P_{1}^{\dagger}\left(I+P_{1}+Q_{1}\right)^{\dagger} G \oplus \mathbf{0} \oplus \mathbf{0}$

$$
=P_{1}^{\dagger}\left(I+P_{1}+Q_{1}\right)^{\dagger} G Q_{1} Q_{1}^{\dagger} G \text {. }
$$

and

$$
\begin{aligned}
\left(P_{1}+Q_{1}\right)\left(P_{1}+Q_{1}\right)^{\dagger} G & =\left(P_{1}+Q_{1}\right) P_{1}^{\dagger}\left(I+P_{1}^{\dagger} Q_{1}\right)^{\dagger} G Q_{1} Q_{1}^{\dagger} \\
& =(P+Q) P^{\dagger}\left(I+P^{\dagger} Q\right)^{\dagger} G Q Q^{\dagger} G .
\end{aligned}
$$

Hence we arrive at

$$
\begin{aligned}
(P+Q)^{\dagger} G= & P^{\dagger}\left(I+P^{\dagger} Q\right)^{\dagger} G Q Q^{\dagger} G+\left(I-Q Q^{\dagger} G\right)\left[\sum_{n=0}^{\infty}\left(P^{\dagger}\right)^{n+1}(-Q)^{n}\right] G+ \\
& {\left[\sum_{n=0}^{\infty}\left(Q^{\dagger}\right)^{n+1}(-P)^{n}\right] G\left(I-P P^{\dagger} G\right) }
\end{aligned}
$$

Now

$$
\begin{gathered}
P^{\dagger}\left(I+P^{\dagger} Q\right)^{\dagger} G Q Q^{\dagger} G=P P^{\dagger}\left(I+P^{\dagger} Q\right)^{\dagger} G Q Q^{\dagger} G+Q P^{\dagger}\left(I+P^{\dagger} Q\right)^{\dagger} G Q Q^{\dagger} G \\
=\left(P P^{\dagger}+Q P^{\dagger}\right)\left(I+P^{\dagger} Q\right)^{\dagger} G Q Q^{\dagger} G \\
\left(I-Q Q^{\dagger} G\right)\left[\sum_{n=0}^{\infty}(-Q)^{n}\left(P^{\dagger}\right)^{n+1}\right] \begin{aligned}
G= & P\left(I-Q Q^{\dagger} G\right) P^{\dagger}\left(I+P^{\dagger} Q\right)^{\dagger} G \cdot G+ \\
& Q P^{\dagger}\left(I-Q Q^{\dagger} G\right)\left(I+P^{\dagger} Q\right)^{\dagger} G \cdot G \\
= & \left(I-Q Q^{\dagger} G\right) P P^{\dagger}\left(I+P^{\dagger} Q\right)+ \\
& Q P^{\dagger}\left(I-Q Q^{\dagger} G\right)\left(I+P^{\dagger} Q\right)^{\dagger} \\
= & \left(I-Q Q^{\dagger} G\right)\left(P P^{\dagger}+Q P^{\dagger}\right)\left(I+P^{\dagger} Q\right)^{\dagger} \cdot(1.7)
\end{aligned}
\end{gathered}
$$


Similarly,

$$
\begin{aligned}
& {\left[\sum_{n=0}^{\infty}(-P)^{n}\left(Q^{\dagger}\right)^{n+1}\right] G\left(I-P P^{\dagger} G\right)=} P Q^{\dagger}\left(I+Q^{\dagger} P\right)^{\dagger} G \cdot G\left(I-P P^{\dagger} G\right)+ \\
& Q Q^{\dagger}\left(I+Q^{\dagger} P\right)^{\dagger} G \cdot G \cdot\left(I-P P^{\dagger} G\right) \\
&=\left(I+Q^{\dagger} P\right)\left[P Q^{\dagger}+Q Q^{\dagger}\right]\left(I-P P^{\dagger} G\right) \\
&=\left(P P^{\dagger}+Q P^{\dagger}\right)\left(I+P^{\dagger} Q\right)^{\dagger} G Q Q^{\dagger} G+ \\
&\left(I-Q Q^{\dagger} G\right)\left(P P^{\dagger}+Q P^{\dagger}\right)\left(I+P^{\dagger} Q\right)+ \\
&\left(I+Q^{\dagger} P\right)\left(P Q^{\dagger}+Q Q^{\dagger}\right)\left(I-P P^{\dagger} G\right) \\
&(P+Q)(P+Q)^{\dagger} G=\left(I+P^{\dagger} Q\right)^{\dagger} G\left(P P^{\dagger}+Q P^{\dagger}\right)\left[I+\left(Q Q^{\dagger} G\right)\right]+ \\
&\left(I+Q^{\dagger} P\right)^{\dagger}\left(P Q^{\dagger}+Q Q^{\dagger}\right)\left(I-P P^{\dagger} G\right)
\end{aligned}
$$

\section{Corollary 2.2:}

If $P$ and $Q$ are GD-invertible in Minkowski space $\mathscr{M}$ and $P Q=0$, then $(P+Q)$ is GD-invertible in $\mathscr{M}$ and $(P+Q)^{\dagger} G=P+(I-G) P+Q(I-G)$.

Proof:

$$
\begin{aligned}
(P+Q)^{\dagger} G= & P^{\dagger}\left(I+P^{\dagger} Q\right)^{\dagger} G Q Q^{\dagger} G+\left(I-Q Q^{\dagger} G\right)\left[\sum_{n=0}^{\infty}(-Q)^{n}\left(P^{\dagger}\right)^{n+1}\right] G+ \\
& {\left[\sum_{n=0}^{\infty}(-P)^{n}\left(Q^{\dagger}\right)^{n+1}\right] G\left(I-Q Q^{\dagger} G\right) }
\end{aligned}
$$

Since $P Q=Q P=0$, so we have $P^{\dagger}\left(P^{\dagger} Q\right)^{\dagger} G Q Q G=P+0=P$

$$
\begin{gathered}
\left(I-Q Q^{\dagger} G\right)\left[\sum_{n=0}^{\infty}(-Q)^{n}\left(P^{\dagger}\right)^{n+1}\right] G=(1-G) P \\
{\left[\sum_{n=0}^{\infty}(-P)^{n}\left(Q^{\dagger}\right)^{n+1}\right] G\left(I-P P^{\dagger} G\right)=Q(I-G)}
\end{gathered}
$$

Therefore $(P+Q)^{\dagger} G=P+(I-G) P+Q(I-G)$.

\section{Corollary 2.3:}

Let $P, Q \in B(X)$ be GD-Invertible such that $P Q-Q P$ and $\left(I+P^{\dagger} Q\right) G$ is GD-Invertible in Minkowski space. If $P Q=Q P=0$, then $P^{\dagger} Q=Q^{\dagger} P=0$ and $(P+Q)^{\dagger} G=P^{\dagger}+P^{\dagger}(I-G)+(I-G) Q^{\dagger}$.

\section{Proof:}

$$
\begin{gathered}
(P+Q)^{\dagger} G=P^{\dagger}\left(I+P^{\dagger} Q\right)^{\dagger} G Q Q^{\dagger} G+\left(I-Q Q^{\dagger} G\right)\left[\sum_{n=0}^{\infty}\left(P^{\dagger}\right)^{n+1}(-Q)^{n}\right] G+ \\
{\left[\sum_{n=0}^{\infty}\left(Q^{\dagger}\right)^{n+1}(-P)^{n}\right] G\left(I-P P^{\dagger} G\right)} \\
P^{\dagger}\left(I+P^{\dagger} Q\right)^{\dagger} G Q Q^{\dagger} G=P^{\dagger}(I+0) G Q Q^{\dagger} G=P^{\dagger} G Q Q^{\dagger} G .
\end{gathered}
$$

\section{Corollary 2.4:}

If $Q$ is quasi-Nilpotent, then $(P+Q)^{\dagger} G=P^{\dagger}+Q^{\dagger}\left(I-P P^{\dagger} G\right)$. 
Proof:

Since $Q$ is Quasi Nilpotent

$$
\begin{aligned}
& P^{\dagger}\left(I+P^{\dagger} Q\right)^{\dagger} G Q Q^{\dagger} G=0 \\
&\left(I-Q Q^{\dagger} G\right)\left[\sum_{n=0}^{\infty}(-Q)^{n}\left(P^{\dagger}\right)^{n+1}\right] G=\left(I-Q Q^{\dagger} G\right)\left[(-Q)^{0}\left(P^{\dagger}\right)^{0+1}\right. \\
&\left.+(-Q)^{1}\left(P^{\dagger}\right)^{1+1}+\cdots\right] G \\
&=\left(I-Q Q^{\dagger} G\right)\left[P^{\dagger} Q P^{\dagger^{2}}+Q^{2} P^{\dagger^{3}}-\cdots\right] G \\
&=\left(I-Q Q^{\dagger} G\right)\left[P^{\dagger}\left(I-Q P^{\dagger}+Q^{2} P^{\dagger^{2}}-\cdots\right)\right] G \\
&=\left(I-Q Q^{\dagger} G\right)\left[P^{\dagger}\left(I+Q P^{\dagger}\right)^{\dagger} G \cdot G\right] \\
&=(I-0) P^{\dagger}(I+0) \\
&= P^{\dagger}
\end{aligned}
$$

$\left[\sum_{n=0}^{\infty}\left(Q^{\dagger}\right)^{n+1}(-P)^{n}\right] G\left(I-P P^{\dagger} G\right)=\left[\left(Q^{\dagger}\right)^{0+1}(-P)^{0}+\left(Q^{\dagger}\right)^{1+1}(-P)^{1}\right.$

$$
\begin{aligned}
& \left.+\left(Q^{\dagger}\right)^{2+1}(-P)^{2}+\cdots\right] G\left(I-P P^{\dagger} G\right) \\
& =\left[Q^{\dagger}-Q^{\dagger^{2}} P+Q^{\dagger^{3}} P^{2}-\cdots\right] G\left(I-P P^{\dagger} G\right) \\
& =Q^{\dagger}\left(I-Q^{\dagger} P+Q^{\dagger^{2}} P^{2}-\cdots\right) G\left(I-P P^{\dagger} G\right) \\
& =Q^{\dagger}\left(I+Q^{\dagger} P\right)^{\dagger} G \cdot G \cdot\left(I-P P^{\dagger} G\right) \\
& =Q^{\dagger}\left(I+P^{\dagger} Q\right)\left(I-P P^{\dagger} G\right) \\
& =Q^{\dagger}\left(I-P P^{\dagger} G\right)
\end{aligned}
$$

Therefore $(P+Q)^{\dagger} G=P^{\dagger}+Q^{\dagger}\left(I-P P^{\dagger} G\right)$

\section{Corollary 2.5:}

If $Q^{k}=0$, then

$$
\begin{aligned}
(P+Q)^{\dagger} G= & P^{\dagger}\left(I+P^{\dagger} Q\right)^{\dagger} G Q Q^{\dagger} G+\left(I-Q Q^{\dagger} G\right)\left[\sum_{n=0}^{k-1}(-Q)^{n}\left(P^{\dagger}\right)^{n+1}\right] G+ \\
& {\left[\sum_{n=0}^{k-1}\left(Q^{\dagger}\right)^{n+1}(-P)^{n}\right] G\left(I-P P^{\dagger} G\right) }
\end{aligned}
$$

Proof:

$$
\begin{aligned}
& P^{\dagger}\left(I+P^{\dagger} Q\right)^{\dagger} G Q Q^{\dagger} G=P^{\dagger}\left(I+P^{\dagger} Q\right)^{\dagger} G Q Q^{\dagger} G \\
& \left(I-Q Q^{\dagger} G\right)\left[\sum_{n=0}^{\infty}(-Q)^{n}\left(P^{\dagger}\right)^{n+1}\right] G=\left(I-Q Q^{\dagger} G\right)\left[(-Q)^{0}\left(P^{\dagger}\right)^{0+1}\right. \\
& \left.+(-Q)^{1}\left(P^{\dagger}\right)^{1+1}+\cdots\right] G \\
& =\left(I-Q Q^{\dagger} G\right)\left[P^{\dagger}-Q P^{\dagger^{2}}+Q^{2} P^{\dagger^{3}}-\cdots\right] G \\
& =\left(I-Q Q^{\dagger} G\right)\left[P^{\dagger}-Q P^{\dagger^{2}}+Q^{2} P^{\dagger^{3}}-\cdots 0\right] G \\
& =\left(I-Q Q^{\dagger} G\right)\left[P^{\dagger}\left(I+Q P^{\dagger}\right)^{\dagger}\right] G \cdot G \\
& =\left(I-Q Q^{\dagger} G\right)\left[P^{\dagger}\left(I+Q P^{\dagger}\right)^{\dagger}\right] \\
& {\left[\sum_{n=0}^{\infty}\left(Q^{\dagger}\right)^{n+1}(-P)^{n}\right] G\left(I-P P^{\dagger} G\right)=\left[\left(Q^{\dagger}\right)^{0+1}(-P)^{0}+\left(Q^{\dagger}\right)^{1+1}(-P)^{1}+\right.} \\
& \left.\left(Q^{\dagger}\right)^{2+1}(-P)^{2}-\cdots\right] G\left(I-P P^{\dagger} Q\right) \\
& =\left[Q^{\dagger}-\left(Q^{\dagger}\right)^{2} P+\left(Q^{\dagger}\right)^{3} P^{2}-\cdots\right] G\left(I-P P^{\dagger} G\right) \\
& =Q^{\dagger}\left[\left(I+Q^{\dagger} P\right)^{\dagger}\right]\left(I-P P^{\dagger} G\right) \\
& =P^{\dagger}\left(I+P^{\dagger} Q\right)^{\dagger} G Q Q^{\dagger} G\left(I-Q Q^{\dagger}\right)\left[P+\left(I+Q P^{\dagger}\right)^{\dagger}\right] \\
& +Q^{\dagger}\left(I+Q^{\dagger} P\right)^{\dagger}\left(I-P P^{\dagger} Q\right)
\end{aligned}
$$

Therefore $(P+Q)^{\dagger} G=\left(I+P^{\dagger} Q\right)^{\dagger}\left[P^{\dagger} G Q Q^{\dagger} G+P^{\dagger}\left(I-Q Q^{\dagger} G\right)+\right.$

$$
\left.P^{\dagger}\left(I-Q Q^{\dagger} G\right)+Q^{\dagger}\left(I-P P^{\dagger} G\right)\right]
$$




\section{Corollary 2.6:}

If $Q^{2}=0,(P+Q)^{\dagger} G=P^{\dagger}\left(I+P^{\dagger} Q\right) G Q Q^{\dagger} G+G+$

$$
\begin{aligned}
& \left(I-Q Q^{\dagger} G\right)\left[\sum_{n=0}^{\infty}(-Q)^{n}\left(P^{\dagger}\right)^{n+1}\right] G+ \\
& {\left[\sum_{n=0}^{\infty}\left(Q^{\dagger}\right)^{n+1}(-P)^{n}\right] G\left(I-P P^{\dagger} G\right)}
\end{aligned}
$$

Proof:

$$
\begin{aligned}
& P^{\dagger}\left(I+P^{\dagger} Q\right)^{\dagger} G Q Q^{\dagger} G=P^{\dagger}\left(I+P^{\dagger} Q\right)^{\dagger} G Q Q^{\dagger} G \\
& \left(I-Q Q^{\dagger} G\right)\left[\sum_{n=0}^{\infty}(-Q)^{n}\left(P^{\dagger}\right)^{n+1}\right] G=\left(I-Q Q^{\dagger} G\right)\left[(-Q)^{0}\left(P^{\dagger}\right)^{0+1}\right. \\
& \left.+(-Q)^{1}\left(P^{\dagger}\right)^{1+1}+(-Q)^{2}\left(P^{\dagger}\right)^{2+1}+\cdots\right] G \\
& =\left(I-Q Q^{\dagger} G\right)\left[P^{\dagger}-Q\left(P^{\dagger}\right)^{2}+Q^{2}\left(P^{\dagger}\right)^{3}-\cdots\right] G \\
& =\left(I-Q Q^{\dagger} G\right) P^{\dagger}\left(I-Q P^{\dagger}\right) \\
& {\left[\sum_{n=0}^{\infty}\left(Q^{\dagger}\right)^{n+1}(-P)^{n}\right] G\left(I-P P^{\dagger} G\right)=\left[\left(Q^{\dagger}\right)^{0+1}(-P)^{0}+\left(Q^{\dagger}\right)^{1+1}(-P)^{1}\right.} \\
& \left.+\left(Q^{\dagger}\right)^{2+1}(-P)^{2}-\cdots\right] G\left(I-P P^{\dagger} G\right) \\
& =\left[Q^{\dagger}-Q^{\dagger^{2}} P+Q^{\dagger^{3}} P^{2}-\cdots\right] G\left(I-P P^{\dagger} G\right) \\
& =Q^{\dagger}\left(I+Q^{\dagger} P\right)^{\dagger} G \cdot G \cdot\left(I-P P^{\dagger} G\right) \\
& =Q^{\dagger}\left(I+Q^{\dagger} P\right)^{\dagger}\left(I-P P^{\dagger} G\right)
\end{aligned}
$$

Therefore $(P+Q)^{\dagger} G=P^{\dagger}\left(I+P^{\dagger} Q\right)^{\dagger} G Q Q^{\dagger} G+\left(I-Q Q^{\dagger} G\right) P^{\dagger}\left(I-Q P^{\dagger}\right) G$

$$
+Q^{\dagger}\left(I+Q^{\dagger} P\right)^{\dagger}\left(I-P P^{\dagger} G\right)
$$

\section{Corollary 2.7:}

If $Q^{k}=Q(k \geq 3)$, then $Q^{\dagger}=Q^{k-2}$

$(P+Q)^{\dagger} G=P^{\dagger}\left(I+P^{\dagger} Q\right)^{\dagger} G Q Q^{\dagger} G(I-P)^{\dagger} G\left[\sum_{n=0}^{\infty}\left(Q^{\dagger}\right)^{n+1}(-P)^{n}\right] G+$

\section{Proof:}

$$
\left[\sum_{n=0}^{\infty}\left(Q^{\dagger}\right)^{n+1}(-P)^{n}\right] G\left(I-P P^{\dagger} G\right)
$$

$$
\begin{aligned}
P^{\dagger}\left(I+P^{\dagger} Q\right)^{\dagger} G Q Q^{\dagger} G & =P^{\dagger}\left(I+Q^{\dagger} P\right)^{\dagger} G Q^{k-2} Q^{\dagger} G \\
& =P^{\dagger}\left(I+Q^{k-2} P\right) G Q^{k-2} Q^{\dagger} G \\
& =P^{\dagger}\left(I+Q^{k}-Q^{-2} P\right)^{\dagger} G Q^{k-2} Q^{\dagger} G \\
& =P^{\dagger}\left(I+Q \cdot Q^{-2} P\right)^{\dagger} G Q^{k-2} Q^{\dagger} G \\
& =P^{\dagger}\left(I+Q^{-1} P\right)^{\dagger} G \\
& =P^{\dagger}\left(I+Q^{\dagger} G P\right)^{\dagger} G \\
(P+Q)^{\dagger} G & =P^{\dagger}\left(I+P^{\dagger} G Q\right)
\end{aligned}
$$

\section{Corollary 2.8:}

If $Q^{2}=Q$. Then $Q^{\dagger}=Q$ and we have $(P+Q)^{\dagger} G=\left(P^{\dagger}+Q\right) G Q G$ $+(I-Q G)\left[P^{\dagger}-P^{\dagger^{2}} Q(I+P)\right]+Q\left(I+P^{\dagger}\right)\left(I-P P^{\dagger} G\right)$. 


\section{Proof:}

$$
\begin{aligned}
& P^{\dagger}\left(I+P^{\dagger} Q\right)^{\dagger} G Q Q^{\dagger} G=P^{\dagger}\left(I+P^{\dagger} Q\right)^{\dagger} G Q \cdot Q G \quad \text { (since } Q^{\dagger}=Q \text { ) } \\
& =P^{\dagger}\left(I+Q^{\dagger} P\right) G Q^{\dagger} G \\
& =P^{\dagger}(I+Q P) G Q G \quad\left(\text { since } Q^{2}=Q\right) \\
& =\left(P^{\dagger}+Q P P^{\dagger}\right) G Q G \\
& =\left(P^{\dagger}+Q\right) G Q G \\
& \left(I-Q Q^{\dagger} G\right)\left[\sum_{n=0}^{\infty}\left(P^{\dagger}\right)^{n+1}(-Q)^{n}\right] G=(I-Q \cdot Q G)\left[\left(P^{\dagger}\right)^{0+1}(-Q)^{0}\right. \\
& \left.+\left(P^{\dagger}\right)^{1+1}(-Q)^{1}+\left(P^{\dagger}\right)^{2+1}(-Q)^{2}+\cdots\right] G \\
& =(I-Q G)\left[P^{\dagger}\left(I+P^{\dagger} Q\right)^{\dagger} G\right] G \\
& =(I-Q G)\left[P^{\dagger}-P^{\dagger^{2}} Q+P^{\dagger^{3}} Q^{2}-P^{\dagger^{4}} Q^{3}+\cdots\right] G \\
& =(I-Q G)\left[P^{\dagger}-P^{\dagger^{2}} Q+P^{\dagger^{3}} Q-P^{\dagger^{4}} Q^{2}+\cdots\right] G \\
& =(I-Q G) P^{\dagger}\left[I-P^{\dagger} Q+P^{\dagger^{2}} Q-P^{\dagger^{3}} Q+\cdots\right] G \\
& =(I-Q G) P^{\dagger}\left[I-P^{\dagger} Q\left(I-P^{\dagger}+P^{\dagger^{2}}-\cdots\right)\right] G \\
& =(I-Q G) P^{\dagger}\left[I-P^{\dagger} Q\left(I+P^{\dagger}\right)^{-1} G\right] \\
& =(I-Q G) P^{\dagger}\left[I-P^{\dagger} Q\left(I+P^{\dagger}\right)^{\dagger} G\right] G \\
& =(I-Q G)\left[P^{\dagger}\left(I-P^{\dagger} Q(I+P)\right)\right] \\
& =(I-Q G)\left[P^{\dagger}-P^{\dagger^{2}} Q(I+P)\right] \\
& {\left[\sum_{n=0}^{\infty}\left(Q^{\dagger}\right)^{n+1}(-P)^{n}\right] G\left(I-P P^{\dagger} G\right)=\left[\sum_{n=0}^{\infty}\left(Q^{\dagger}\right)^{n+1}\left(-P^{n}\right)\right] G\left(I-P P^{\dagger} G\right)} \\
& =\left[Q^{0+1}(-P)^{0}+Q^{1+1}(-P)^{1}+Q^{2+1}(-P)^{2}+\cdots\right] G\left(I-P P^{\dagger} G\right) \\
& =\left[Q-Q^{2} P+Q^{3} P^{2}-\cdots\right] G\left(I-P P^{\dagger} G\right) \\
& =\left[Q-Q P+Q P^{2}-\cdots\right] G\left(I-P P^{\dagger} G\right) \quad\left(\text { since } Q^{2}=Q^{\dagger}=Q\right) \\
& =\left[Q(I+P)^{-1}\right] G\left(I-P P^{\dagger} G\right) \\
& =Q(I+P)^{\dagger} G \cdot G\left(I-P P^{\dagger} G\right) \\
& =Q\left(I+P^{\dagger}\right)\left(I-P P^{\dagger} G\right)
\end{aligned}
$$

Therefore

$$
(P+Q)^{\dagger} G=\left(P^{\dagger}+Q\right) G Q G+(I-Q G)\left[P^{\dagger}-P^{\dagger^{2}} Q(I+P)\right]+Q\left(I+P^{\dagger}\right)\left(I-P P^{\dagger} G\right) .
$$

\section{Corollary 2.9:}

If $P^{2}=P$ and $Q^{2}=Q$, then $I+P Q$ is invertible and $P(I+P Q)^{\dagger} G Q=\frac{1}{2} P Q$ and we have $(P+Q)^{\dagger} G=P^{\dagger}+Q^{\dagger}+(I-G) P^{\dagger}\left(I-P^{\dagger} Q-Q\right)+$

$$
Q^{\dagger}\left(I-Q^{\dagger} P-P\right)(I-G) \text {. }
$$




$$
\begin{aligned}
& =\left[Q^{\dagger}-Q^{\dagger^{2}} P+Q^{\dagger^{3}} P-\cdots\right] G(I-G) \\
& =Q^{\dagger}\left[I-Q^{\dagger} P+Q^{\dagger^{2}} P-\cdots\right] G(I-G) \\
& =Q^{\dagger}\left[I-Q^{\dagger} P\left(I-Q^{\dagger}+Q^{\dagger^{2}}-\cdots\right)\right] G(I-G) \\
& =Q^{\dagger}\left[I-Q^{\dagger} P\left(I+Q^{\dagger}\right)^{\dagger} G\right] G(I-G) \\
& =Q^{\dagger}\left[I-Q^{\dagger} P(I+Q)\right](I-G) \\
& =Q^{\dagger}\left[I-Q^{\dagger} P-Q Q^{\dagger} P\right](I-G) \\
& =Q^{\dagger}\left[I-Q^{\dagger} P-P\right](I-G)
\end{aligned}
$$

Therefore

$$
(P+Q)^{\dagger} G=P^{\dagger}+Q^{\dagger}+(I-G) P^{\dagger}\left(I-P^{\dagger} Q-Q\right)+Q^{\dagger}\left(I-Q^{\dagger} P-P\right)(I-G) .
$$

\section{Theorem 2.10:}

Let $A \in B(X)$ and $B \in B(Y)$ are GD-invertible $C \in B(X, Y)$. Then

$$
\begin{aligned}
M= & \left(\begin{array}{ll}
A & C \\
O & B
\end{array}\right) \text { are GD-invertible and } M^{\dagger}=\left(\begin{array}{cc}
A^{\dagger} & X \\
O & B^{\dagger}
\end{array}\right) \text {, where } \\
X= & \left(A^{\dagger}\right)^{2}\left[\sum_{n=0}^{\infty}\left(A^{\dagger}\right)^{n} C B^{n}\right] G(I-G) \\
& +(I-G)\left[\sum_{n=0}^{\infty} A^{n} C\left(B^{\dagger}\right)^{n}\right] G\left(B^{\dagger}\right)^{2}-A^{\dagger} G C B^{\dagger} G .
\end{aligned}
$$

In [3], it is presented an expression of $(P+Q)^{\dagger} G$ under the condition that $Q$ is quasi-nilpotent such that $P^{\pi} P Q=P^{\pi} Q P$ and $Q=Q P^{\pi}$.

Proof:

$$
\begin{aligned}
& P^{\dagger}\left(I+P^{\dagger} Q\right)^{\dagger} G Q Q^{\dagger} G=P^{\dagger}\left(I+Q^{\dagger} P\right) G Q Q^{\dagger} G \\
& =\left(P^{\dagger}+Q^{\dagger} P P^{\dagger}\right) \cdot I \\
& =P^{\dagger}+Q^{\dagger} \\
& \left(I-Q Q^{\dagger} G\right)\left[\sum_{n=0}^{\infty}\left(P^{\dagger}\right)^{n+1}(-Q)^{n}\right] G=(I-G)\left[\left(P^{\dagger}\right)^{0+1}(-Q)^{0}+\right. \\
& \left.\left(P^{\dagger}\right)^{1+1}(-Q)^{1}+\left(P^{\dagger}\right)^{2+1}(-Q)^{2}+\cdots\right] G \\
& =(I-G)\left[P^{\dagger}-P^{\dagger^{2}} Q+P{\dagger^{3}}^{2}-\cdots\right] G \\
& =(I-G)\left[P^{\dagger}-P^{\dagger^{2}} Q+P^{\dagger^{2}} Q-\cdots\right] G \\
& =(I-G) P^{\dagger}\left[I-P^{\dagger} Q\left(I-P^{\dagger}+P^{\dagger^{2}}-\cdots\right)\right] G \\
& =(I-G) P^{\dagger}\left[I-P^{\dagger} Q\left(I+P^{\dagger}\right)^{\dagger} G \cdot G\right] \\
& =(I-G) P^{\dagger}\left[I-P^{\dagger} Q(I+P)\right] \\
& =(I-G) P^{\dagger}\left[I-P^{\dagger} Q-P Q^{\dagger} Q\right] \\
& =(I-G) P^{\dagger}\left(I-P^{\dagger} Q-Q\right) \\
& {\left[\sum_{n=0}^{\infty}\left(Q^{\dagger}\right)^{n+1}(-P)^{n}\right] G\left(I-P P^{\dagger} G\right)=\left[\left(Q^{\dagger}\right)^{0+1}(-P)^{0}+\left(Q^{\dagger}\right)^{1+1}(-P)^{1}\right.} \\
& \left.+\left(Q^{\dagger}\right)^{2+1}(-P)^{2}+\cdots\right] G(I-G)
\end{aligned}
$$




$$
\begin{aligned}
& =\left[Q^{\dagger}-Q^{\dagger^{2}} P+Q^{\dagger^{3}} P-\cdots\right] G(I-G) \\
& =Q^{\dagger}\left[I-Q^{\dagger} P+Q^{\dagger^{2}} P-\cdots\right] G(I-G) \\
& =Q^{\dagger}\left[I-Q^{\dagger} P\left(I-Q^{\dagger}+Q^{\dagger^{2}}-\cdots\right)\right] G(I-G) \\
& =Q^{\dagger}\left[I-Q^{\dagger} P\left(I+Q^{\dagger}\right)^{\dagger} G\right] G(I-G) \\
& =Q^{\dagger}\left[I-Q^{\dagger} P(I+Q)\right](I-G) \\
& =Q^{\dagger}\left[I-Q^{\dagger} P-Q Q^{\dagger} P\right](I-G) \\
& =Q^{\dagger}\left[I-Q^{\dagger} P-P\right](I-G)
\end{aligned}
$$

Therefore

$$
(P+Q)^{\dagger} G=P^{\dagger}+Q^{\dagger}+(I-G) P^{\dagger}\left(I-P^{\dagger} Q-Q\right)+Q^{\dagger}\left(I-Q^{\dagger} P-P\right)(I-G) .
$$

\section{Theorem 2.10:}

Let $A \in B(X)$ and $B \in B(Y)$ are GD-invertible $C \in B(X, Y)$. Then

$$
\begin{aligned}
M= & \left(\begin{array}{ll}
A & C \\
O & B
\end{array}\right) \text { are GD-invertible and } M^{\dagger}=\left(\begin{array}{cc}
A^{\dagger} & X \\
O & B^{\dagger}
\end{array}\right) \text {, where } \\
X= & \left(A^{\dagger}\right)^{2}\left[\sum_{n=0}^{\infty}\left(A^{\dagger}\right)^{n} C B^{n}\right] G(I-G) \\
& +(I-G)\left[\sum_{n=0}^{\infty} A^{n} C\left(B^{\dagger}\right)^{n}\right] G\left(B^{\dagger}\right)^{2}-A^{\dagger} G C B^{\dagger} G .
\end{aligned}
$$

In [3], it is presented an expression of $(P+Q)^{\dagger} G$ under the condition that $Q$ is quasi-nilpotent such that $P^{\pi} P Q=P^{\pi} Q P$ and $Q=Q P^{\pi}$.

In fact, if we answer that $P^{\pi} Q\left(1-P^{\pi}\right)=0$, instead of $Q$ quasi-nilpotent with $Q=Q P^{\pi}$, we will get a general result.

\section{Theorem 2.11:}

Let $P \in B(X)$ be GD-invertible in Minkowski space $\mathscr{M}$ and $Q \in B(X)$ such that $\left\|Q P^{\dagger}\right\|<1, P^{\pi} Q\left(1-P^{\pi}\right)=0$ and $P^{\pi} P Q=P^{\pi} Q$ is GD-invertible in $m$, then $(P+Q)$ is GD-invertible. In this case,

$$
\begin{aligned}
(P+Q)^{\dagger} G= & \left(I+P^{\dagger} Q\right)^{\dagger} G P^{\dagger} G+\left(I+P^{\dagger} Q\right)^{\dagger} G\left(I-P P^{\dagger} G\right)\left[\sum_{n=0}^{\infty}\left(Q^{\dagger}\right)^{n+1}(-P)^{n}\right] G \\
& +\left[\sum_{n=0}^{\infty}\left(\left(I+P^{\dagger} Q\right)^{\dagger} G P^{\dagger}\right)^{n+2} Q\left(I-P P^{\dagger} G\right)(P+Q)^{n}\right]\left(I-P P^{\dagger} G\right) \\
& \times\left\{I-(P+Q)\left(I-P P^{\dagger} G\right)\left[\sum_{n=0}^{\infty}\left(Q^{\dagger}\right)^{n+1}(-P)^{n}\right] G\right\} .
\end{aligned}
$$

\section{Proof:}

Since $P$ is GD-invertible and $P^{\pi} Q\left(1-P^{\pi}\right)=0, P$ and $Q$ have the form 
$P=\left(\begin{array}{cc}P_{1} & 0 \\ 0 & P_{2}\end{array}\right)$ and $Q=\left(\begin{array}{cc}Q_{1} & Q_{3} \\ 0 & Q_{2}\end{array}\right)$ with respect to the space decomposition $X=N\left(P^{\pi}\right) \oplus R\left(P^{\pi}\right)$, where $P_{1}$ is invertible and $P_{2}$ is quasi-nilpotent.

$\left\|Q P^{\dagger} G\right\|<1$ implies that $I+P^{\dagger} Q$ is invertible.

$P^{\pi} Q P=P^{\dagger} P Q$ implies that $P_{2} Q_{2}=Q_{2} P_{2}$. Since $P^{\pi} Q$ is GD-invertible, $Q_{2}$ is GD-invertible. It follows from Theorem 2.1 that

$\left(P_{2}+Q_{2}\right)^{\dagger} G=\left[\sum_{n=0}^{\infty}\left(Q_{2}^{\dagger}\right)^{n+2}\left(-P_{2}\right)^{2}\right] G$

By Theorem 2.10, $(P+Q)^{\dagger} G$ has the form

$$
\begin{aligned}
(P+Q)^{\dagger} G= & {\left[\begin{array}{cc}
\left(P_{1}+Q_{1}\right)^{\dagger} G & S \\
0 & \sum_{n=0}^{\infty}\left(Q_{2}^{\dagger}\right)^{n+2}\left(-P_{2}\right)^{n}
\end{array}\right], } \\
\text { where } S= & {\left[\sum_{n=0}^{\infty}\left(\left(P_{1}+Q_{1}\right)^{\dagger}\right)^{n+2} G Q_{3}\left(P_{2}+Q_{2}\right)^{n}\right] } \\
& {\left[I-\left(P_{2}+Q_{2}\right)\left(\sum_{n=0}^{\infty}\left(Q_{2}^{\dagger}\right)^{n+1}\left(-P_{2}\right)^{n}\right) G\right] } \\
& -\left(P_{1}+Q_{1}\right)^{\dagger} G Q_{3}\left(\sum_{n=0}^{\infty}\left(Q_{2}^{\dagger}\right)^{n+1}\left(-P_{2}\right)^{n}\right) G .
\end{aligned}
$$

Note that the product and the sum pf $P, Q, P^{\dagger} G$ and $Q^{\dagger} G$ are still the upper triangular operator matrices.

Thus $\left(P_{1}+Q_{1}\right)^{\dagger} G=P_{1}^{\dagger}\left(I+P_{1}^{\dagger} Q\right)^{\dagger} G$

and

$$
\begin{aligned}
& 0 \oplus\left(\sum_{n=0}^{\infty}\left(Q_{2}^{\dagger}\right)^{n+1}\left(-P_{2}\right)^{n}\right) G=P^{\pi}\left[\sum_{n=0}^{\infty}\left(Q^{\dagger}\right)^{n+1}(-P)^{n}\right] G \\
& {\left[\begin{array}{cc}
\left(P_{1}+Q_{1}\right) G & 0 \\
0 & 0
\end{array}\right]+\left[\begin{array}{cc}
\left(P_{1}+Q_{1}\right) G & 0 \\
0 & \left(\sum_{n=0}^{\infty}\left(Q^{\dagger}\right)^{n+1}(-P)^{n}\right) G
\end{array}\right]+\left(\begin{array}{ll}
0 & S \\
0 & 0
\end{array}\right)} \\
& =P^{\dagger}\left(I+P^{\dagger} Q\right)^{\dagger} G+P^{\dagger}\left(I+P^{\dagger} Q\right)^{\dagger} G P^{\pi}\left[\sum_{n=0}^{\infty}\left(Q^{\dagger}\right)^{n+1}(-P)^{n}\right] G+ \\
& {\left[\sum_{n=0}^{\infty}\left(\left(I+P^{\dagger} Q\right)^{\dagger} P^{\dagger}\right)^{n+2} G Q P^{\pi}(P+Q)^{n}\right] P^{\pi}} \\
& {\left[I-(P+Q) P^{\pi}\left(\sum_{n=0}^{\infty}\left(Q^{\dagger}\right)^{n+1}(-P)^{n}\right) G\right]} \\
& -P^{\dagger}\left(I+P^{\dagger} Q\right)^{\dagger} G \cdot G P^{\dagger} Q P^{\pi}\left(\sum_{n=0}^{\infty}\left(Q^{\dagger}\right)^{n+1}(-P)^{n}\right) G
\end{aligned}
$$




$$
\begin{aligned}
& =-P^{\dagger}\left(I+P^{\dagger} Q\right)^{\dagger} G \cdot G P^{\dagger} Q P^{\pi}\left(\sum_{n=0}^{\infty}\left(Q^{\dagger}\right)^{n+1}(-P)^{n}\right) G \\
& +\left(I+P^{\dagger} Q\right)^{\dagger} P^{\dagger} Q P^{\pi}\left(I+Q^{\dagger} P\right)^{\dagger} G \cdot G \quad\left[\because P^{\dagger} Q=0\right] \\
& (P+Q)^{\dagger} G=P^{\dagger}\left(I+P^{\dagger} Q\right)^{\dagger} G+P^{\dagger}\left(I+P^{\dagger} Q\right)^{\dagger} G P^{\pi}\left(\sum_{n=0}^{\infty}\left(Q^{\dagger}\right)^{n+1}(-P)^{n}\right) G \\
& +\left[\sum_{n=0}^{\infty}\left(\left(I+P^{\dagger} Q\right)^{\dagger} P^{\dagger}\right)^{n+2} G Q P^{\pi}(P+Q)^{n}\right] P^{\pi} \\
& {\left[I-(P+Q) P^{\pi}\left(\sum_{n=0}^{\infty}\left(Q^{\dagger}\right)^{n+1}(-P)^{n}\right) G\right] .}
\end{aligned}
$$

\section{Corollary 2.12:}

Let $P \in B(X)$ be GD-invertible and $Q \in B(X)$ such that $\left\|Q P^{\dagger} G\right\|<1$, $P^{\pi} Q\left(1-P^{\pi}\right)=0$ and $P^{\pi} P Q=P^{\pi} Q P$.

1. If $Q P P^{\dagger} G=0$ and $Q$ is quasi-nilpotent, then Theorem 2.11 is simplified to

$$
\begin{aligned}
(P+Q)^{\dagger} G= & P^{\dagger}\left(I+P^{\dagger} Q\right)^{\dagger} G+P^{\dagger}\left(I+P^{\dagger} Q\right)^{\dagger} G P^{\pi}\left(\sum_{n=0}^{\infty}\left(Q^{\dagger}\right)^{n+1}(-P)^{n}\right) G \\
& +\left[\sum_{n=0}^{\infty}\left(\left(I+P^{\dagger} Q\right)^{\dagger} P^{\dagger}\right)^{n+2} G Q P^{\pi}(P+Q)^{n}\right] \\
& P^{\pi}\left[I-(P+Q) P^{\pi}\left(\sum_{n=0}^{\infty}\left(Q^{\dagger}\right)^{n+1}(-P)^{n}\right) G\right] \\
& P^{\pi}\left[I-(P+Q) P^{\pi}\left(\sum_{n=0}^{\infty}\left(Q^{\dagger}\right)^{n+1}(-P)^{n}\right) G\right]
\end{aligned}
$$

$P^{\dagger}\left(I+P^{\dagger} Q\right)^{\dagger} G=P^{\dagger}(I+0)^{\dagger} G \quad$ (since $Q$ is quasi-nilpotent)

$$
=P^{\dagger} G
$$

$P^{\dagger}\left(I+P^{\dagger} Q\right)^{\dagger} G P^{\pi}\left(\sum_{n=0}^{\infty}\left(Q^{\dagger}\right)^{n+1}(-P)^{n}\right) G=P^{\dagger}(I+0)^{\dagger} G P^{\pi} G^{\dagger}\left(I+Q^{\dagger} P\right)^{\dagger} G \cdot G$

$$
\begin{aligned}
& =P^{\dagger} G P^{\pi} Q^{\dagger}\left(I+P^{\dagger} Q\right) \\
& =P^{\dagger} G P^{\pi}\left(Q^{\dagger}+P^{\dagger}\right)
\end{aligned}
$$

$$
\begin{aligned}
& {\left[\sum_{n=0}^{\infty}\left(\left(I+P^{\dagger} Q\right)^{\dagger} P^{\dagger}\right)^{n+2} G Q P^{\pi}(P+Q)^{n}\right]} \\
& P^{\pi}\left[I-(P+Q) P^{\pi}\left(\sum_{n=0}^{\infty}\left(Q^{\dagger}\right)^{n+1}(-P)^{n}\right) G\right] \\
& =\left[\sum_{n=0}^{\infty}\left(P^{\dagger}\right)^{n+2} G Q(P+Q)^{n}\right](I-0)\left[I-P Q^{\dagger}\left(I+Q^{\dagger} P\right)^{\dagger} G \cdot G\right]
\end{aligned}
$$

$\left[\sum_{n=0}^{\infty}\left(P^{\dagger}\right)^{n+2} G Q(P+Q)^{n}\right]\left[I-P Q^{\dagger}\left(I+P^{\dagger} Q\right)\right]$

(since $Q P^{\pi}=Q$ ) 


$$
\begin{gathered}
=\left[\sum_{n=0}^{\infty}\left(P^{\dagger}\right)^{n+2} G Q(P+Q)^{n}\right]\left[I-P Q^{\dagger}(I)\right] \\
=\left[\sum_{n=0}^{\infty}\left(P^{\dagger}\right)^{n+2} G Q(P+Q)^{n}\right]\left[I-P Q^{\dagger}\right] \\
\therefore(P+Q)^{\dagger} G=P^{\dagger} G+P^{\dagger} G P^{\pi}\left(Q^{\dagger}+P^{\dagger}\right)+\left[\sum_{n=0}^{\infty}\left(P^{\dagger}\right)^{n+2} G Q(P+Q)^{n}\right]\left(I-P G^{\dagger}\right)
\end{gathered}
$$

2. If $P^{\pi} Q=Q P^{\pi}, \sigma\left(P^{\pi} Q\right)=0$,

$$
\begin{gathered}
(P+Q)^{\dagger} G=P^{\dagger}\left(I+P^{\dagger} Q\right)^{\dagger} G \\
\left(I+P^{\dagger} Q\right)^{\dagger} G P^{\pi}\left(\sum_{n=0}^{\infty}\left(Q^{\dagger}\right)^{n+1}(-P)^{n}\right) G=0 \\
{\left[\sum_{n=0}^{\infty}\left(\left(I+P^{\dagger} Q\right)^{\dagger} P^{\dagger}\right)^{n+2} G Q P^{\pi}(P 6 Q)^{n}\right]} \\
P^{\pi}\left[I-(P+Q) P^{\pi}\left(\sum_{n=0}^{\infty}\left(Q^{\dagger}\right)^{n+2}(-P)^{n}\right) G\right]=0
\end{gathered}
$$

Therefore

$$
(P+Q)^{\dagger} G=P^{\dagger}\left(I+P^{\dagger} Q\right)^{\dagger} G .
$$

\section{Theorem 2.13:}

Let $P$ and $Q \in B(X)$ be GD-invertible. Let $F$ be an idempotent such that $F P=P F,(I-F) Q F=0,(P Q-Q P) F=0$ and $(I-F)(P Q-Q P)=0$. If $(P+Q) F$ and $(I-F)(P+Q)$ are GD-invertible, than $(P+Q)$ is GD-invertible and $(P+Q)^{\dagger} G=\sum_{n=0}^{\infty} \Delta^{n+2} F Q(I-F)(P+Q)^{n} F Q(I-F) \Delta^{n+2}$

$$
+(I-\Delta F Q)(I-F) \Delta+\Delta F,
$$

where $\Delta=P^{\dagger}\left(I+P^{\dagger} Q\right)^{\dagger} G Q Q^{\dagger} G+\left(I-Q Q^{\dagger} G\right)\left(\sum_{n=0}^{\infty}(-Q)^{n}\left(P^{\dagger}\right)^{n+1}\right) G$

$$
+\left(\sum_{n=0}^{\infty}\left(Q^{\dagger}\right)^{n+1}(-P)^{n}\right) G\left(I-P P^{\dagger} G\right)
$$

\section{Proof:}

Since $F^{2}=F$, we have $F=I \oplus 0$ with respect to space decomposition $X=R(F) \oplus N(F)$. From $F P=P F$ and $(I-F) Q F=0$, we know that

$$
P=\left(\begin{array}{cc}
P_{1} & 0 \\
0 & P_{2}
\end{array}\right) ; Q=\left(\begin{array}{cc}
Q_{1} & Q_{3} \\
0 & Q_{2}
\end{array}\right)
$$

Since $(P+Q) F$ and $(I-F)(P+Q)$ are GD-invertible $P_{i}+Q_{i}(i=1,2)$ are GD-invertible. 
Hence $(P+Q)^{\dagger} G=\left(\begin{array}{cc}\left(P_{1}+Q_{1}\right)^{\dagger} G & X \\ 0 & \left(P_{2}+Q_{2}\right)^{\dagger} Q\end{array}\right)$ where

$$
\begin{aligned}
X= & {\left[\sum_{n=0}^{\infty}\left(\left(P_{1}+Q_{1}\right)^{\dagger} G\right)^{n+2} Q_{3}\left(P_{2}+Q_{2}\right)^{n}\right]\left[I-\left(P_{2}+Q_{2}\right)\left(P_{2}+Q_{2}\right)^{\dagger} G\right] } \\
& +\left[I-\left(P_{1}+Q_{1}\right)\left(P_{1}+Q_{1}\right)^{\dagger} G\right]\left[\sum_{n=0}^{\infty}\left(P_{1}+Q_{1}\right)^{n} Q_{3}\left(\left(P_{2}+Q_{2}\right)^{\dagger} G\right)^{n+2}\right] \\
& -\left(\left(P_{1}+Q_{1}\right)^{\dagger} G\right) Q_{3}\left(P_{2}+Q_{2}\right)^{\dagger} G .
\end{aligned}
$$

By Theorem 2.10, from $(P Q-Q P) F=0$ and $(I-F)(P Q-Q P)=0$.

We know that $P_{i} Q_{i}=Q_{i} P_{i}(i=1,2)$, note that $P, Q, P^{\dagger} G$ and $A^{\dagger} G$ are all the upper triangular operator matrices by Theorem 2.1. It shows that

$$
\begin{aligned}
\left(P_{1}+Q_{1}\right)^{\dagger} G \oplus 0= & P_{1}^{\dagger}\left(I+P_{1}^{\dagger} Q_{1}\right)^{\dagger} G Q_{1} Q_{1}^{\dagger} G \oplus 0+ \\
& \left(I-Q_{1} Q_{1}^{\dagger} G\right)\left(\sum_{n=0}^{\infty}\left(-Q_{1}^{\dagger}\right)^{n}\left(P_{1}^{\dagger}\right)^{n+1}\right) G \oplus 0+ \\
& \left(\sum_{n=0}^{\infty}\left(Q_{1}^{\dagger}\right)^{n+1}\left(-P_{1}\right)^{n}\right)\left(I-P_{1} P_{1}^{\dagger} G\right) \oplus 0 \\
= & P^{\dagger}\left(I P^{\dagger} Q\right)^{\dagger} G Q Q^{\dagger} G F+ \\
& \left(I-Q Q^{\dagger} G\right)\left(\sum_{n=0}^{\infty}(-Q)^{n}\left(P^{\dagger}\right)^{n+1}\right) G F+ \\
& \left(\sum_{n=0}^{\infty}\left(Q^{\dagger}\right)^{n+1}(-P)^{n}\right) G\left(I-P P^{\dagger} G\right) F \\
\left(P_{1}+Q_{1}\right)^{\dagger} G \oplus 0= & \Delta F
\end{aligned}
$$

Where $\Delta$ is defined in Equation (1.9).

Similarly we can prove

$$
\begin{aligned}
& 0 \oplus\left(P_{2}+Q_{2}\right)^{\dagger} G= 0 \oplus P_{2}^{\dagger}\left(I+P_{2}^{\dagger} Q_{2}\right)^{\dagger} G Q_{2} Q_{2}^{\dagger}\left(I-Q_{2} Q_{2}^{\dagger} G\right) \\
&\left(\sum_{n=0}^{\infty}\left(-Q_{2}\right)^{n}\left(P_{2} \dagger\right)^{n+1}\right) G+ \\
&\left(\sum_{n=0}^{\infty}\left(Q_{2}^{\dagger}\right)^{n+1}\left(-P_{2}\right)^{n}\right) G\left(I P_{2} P_{2}^{\dagger} G\right) \\
&\left.0 \oplus P_{2}^{\dagger}\left(I+P_{2}^{\dagger} Q_{2}\right)^{\dagger}=(I-F) P^{\dagger}\left(I+P^{\dagger} Q\right)^{\dagger} G Q Q^{\dagger} G \quad \text { (since } F=I \oplus 0\right] \\
& 0 \oplus\left(I-Q_{2} Q_{2}^{\dagger} G\right)\left(\sum_{n=0}^{\infty}\left(-Q_{2}\right)^{n}\left(P_{2}^{\dagger}\right)^{n+1}\right) G=(I-F)\left(I-Q Q^{\dagger} G\right)
\end{aligned}
$$




$$
\begin{aligned}
& \left(\sum_{n=0}^{\infty}(-Q)^{n}\left(P^{\dagger}\right)^{n+1}\right) G \\
& 0 \oplus\left(\sum_{n=0}^{\infty}\left(Q_{2}^{\dagger}\right)^{n+1}\left(-P_{2}\right)^{n}\right) G\left(I-P_{2} P_{2}^{\dagger} G\right)=(I-F)\left(\sum_{n=0}^{\infty}\left(Q^{\dagger}\right)^{n+1}(-P)^{n}\right) \\
& G\left(I-P P^{\dagger} G\right) \\
& 0 \oplus\left(P_{2}+Q_{2}\right)^{\dagger} G=(I-F) P^{\dagger}\left(I+P^{\dagger} Q\right)^{\dagger} G Q Q^{\dagger} G+(I-F)\left(I-Q Q^{\dagger} G\right) \\
& \left(\sum_{n=0}^{\infty}(-Q)^{n}\left(P^{\dagger}\right)^{n+1}\right) G+(I-F)\left(\sum_{n=0}^{\infty}\left(Q^{\dagger}\right)^{n+1}\left(-P^{\dagger}\right)^{n}\right) G\left(I-P P^{\dagger} G\right) \\
& =(I-F)\left\{P^{\dagger}\left(I+P^{\dagger} Q\right)^{\dagger} G Q Q^{\dagger} G+\left(I-Q Q^{\dagger} G\right)\left[\sum_{n=0}^{\infty}(-Q)^{n}\left(P^{\dagger}\right)^{n+1}\right] G\right. \\
& \left.+\left[\sum_{n=0}^{\infty}\left(Q^{\dagger}\right)^{n+1}(-P)^{n}\right) G\left(I-P P^{\dagger} G\right)\right\} \\
& =(I-F) \Delta
\end{aligned}
$$

We observe that,

$$
\begin{aligned}
& \left(\begin{array}{cc}
0 & \left(\left(P_{1}+Q_{1}\right)^{\dagger} G\right)^{n+2} Q_{3}\left(P_{2}+Q_{2}\right)^{n}\left(I-\left(P_{2}+Q_{2}\right)\left(P_{2}+Q_{2}\right)+G\right) \\
0 & 0
\end{array}\right) \\
= & {\left[\because\left(P_{1}+Q_{1}\right)^{\dagger} G=\Delta F\right] } \\
= & {\left[\Delta^{n+2} F Q(I-F)(P+Q)^{n+2} F Q(I-F)(P+Q)^{n}\right][I-(P+Q)(I-F) \Delta] }
\end{aligned}
$$

(since $F$ is idempotent]

with

$$
\begin{array}{cc}
\left(\begin{array}{cc}
0 & {\left[I-\left(P_{1}+Q_{1}\right)\left(P_{1}+Q_{1}\right)^{\dagger} G\right]\left[\left(P_{1}+Q_{1}\right)^{n} Q_{3}\left(\left(P_{2}+Q_{2}\right)^{\dagger} G\right)^{n+2}\right]} \\
0 & 0
\end{array}\right) \\
\quad=[I-(P+Q) \Delta F]\left[(P+Q)^{n} F Q(I-F)((I-F) \Delta)^{n+2}\right] \\
\quad=(I-(P+Q) \Delta)(P+Q)^{n} F^{2} Q(I-F) \Delta^{n+2} \quad \text { (since } F \text { is idempotent) } \\
\quad=(I-(P+Q) \Delta)(P+Q)^{n} F Q(I-F) \Delta^{n+2}
\end{array}
$$

and 


$$
\begin{aligned}
\left(\begin{array}{cc}
0 & -\left(P_{1}+Q_{1}\right)+G Q_{3}\left(P_{2}+Q_{2}\right)+G \\
0 & \left(P_{2}+Q_{2}+G\right)
\end{array}\right) & =\left(\begin{array}{cc}
0 & -(\Delta F) F Q(I-F) \Delta \\
0 & (I-F) \Delta
\end{array}\right) \\
& =-\Delta F^{2} Q(I-F) \Delta+(I-F) \Delta \\
& \quad(\text { since } F \text { is idempotent) } \\
& =(I-F) \Delta[I-\Delta F Q]
\end{aligned}
$$

Hence

$$
\begin{aligned}
(P+Q)^{\dagger}= & \Delta^{n+2} F Q(I-F)(P+Q)^{n}[I-(P+Q) \Delta]+[I-(P+Q) \Delta](P+Q)^{n} F Q \\
& (I-F) \Delta^{n+2}+(I-F) \Delta(I-\Delta F Q)+\Delta F
\end{aligned}
$$

Therefore

$$
\begin{aligned}
(P+Q)^{\dagger} G= & \sum_{n=0}^{\infty} \Delta^{n+2} F Q(I-F)(P+Q)^{n}(I-(P+Q) \Delta)+ \\
& {[I-(P+Q) \Delta](P+Q)^{n} F Q(I-F) \Delta^{n+2}+} \\
& (I-F) \Delta(I-\Delta F Q)+\Delta F .
\end{aligned}
$$

Hence Proved.

\section{III.Special Cases}

Let us use Theorem 2.13 to analyze some interesting special perturbations of linear operators. We thereby extend earlier work by several authors $[4,6,7$, 12, 13] and partially solve a problem posed in 1975 by Campbell and Meyer, who consider it difficult to establish the norm estimates for the perturbation of the Drazin inverse.

Case (i): If $Q F=0$, then $\Delta F=P^{\dagger} G F, Q \Delta F=0$, thus Theorem 2.13 reduces

to

$$
\begin{aligned}
(P+Q)^{\dagger} G= & \sum_{n=0}^{\infty} \Delta^{n+2} F Q(I-F)(P+Q)^{n}(I-F)[I-(P+Q) \Delta]+[I-(P+Q) \Delta] F \\
& \sum_{n=0}^{\infty}(P+Q)^{n} F Q(I-F) \Delta^{n+2}+(I-\Delta F Q)(I-F) \Delta+\Delta F \\
(P+Q)^{\dagger} G= & \sum_{n=0}^{\infty}(P+Q)^{n+2} F Q(P+Q)^{n}[I-(P+Q) \Delta]+\left(I-P P^{\dagger} G\right)
\end{aligned}
$$




$$
\begin{aligned}
& \sum_{n=0}^{\infty}(P+Q)^{n} F Q \Delta^{n+2}-P^{\dagger} G F Q \Delta+(I-F) \Delta+P^{\dagger} G F \\
& (P+Q)^{\dagger} G=\sum_{n=0}^{\infty} \Delta^{n+2} F Q(I-F)(P+Q)^{n}(I-F)[I-(P+Q) \Delta]+[I-(P+Q) \Delta] F \\
& \sum_{n=0}^{\infty}(P+Q)^{n} F Q(I-F) \Delta^{n+2}+(I-\Delta F Q)(I-F) \Delta+\Delta F \\
& \Delta F=0, \Delta F=P^{\dagger} G F, \Delta=P^{\dagger} G, Q \Delta F=0 \\
& (P+G)^{\dagger} G=\sum_{n=0}^{\infty} F Q(P+Q)^{n}(I-(P+Q) \Delta) \\
& {[I-(P+Q) \Delta]=\sum_{n=0}^{\infty}(P+Q)^{n} F Q(I-F) \Delta^{n+2}=(I-(P \Delta F+Q \Delta F))} \\
& \sum_{n=0}^{\infty}(P+Q)^{n} F Q \Delta^{n+2} \\
& =\left(I-P P^{\dagger} G\right) \sum_{n=0}^{\infty}(P+Q)^{n} F Q \Delta^{n+2} \\
& (I-\Delta F Q)(I-F) \Delta+\Delta F=(\Delta-\Delta F Q \Delta)(I-F) \\
& =\Delta-\Delta F Q \Delta-\Delta F+\Delta F Q \Delta F \\
& =\Delta-\Delta F Q \Delta-\Delta F+O \\
& =-\Delta F Q \Delta+\Delta-\Delta F \\
& =P^{\dagger} G F Q \Delta+(I-F) \Delta+\Delta F \\
& =P^{\dagger} G F Q \Delta+(I-F) \Delta+P^{\dagger} G F
\end{aligned}
$$

Therefore $(P+Q)^{\dagger} G=\sum_{n=0}^{\infty}\left(P^{\dagger} G\right)^{n+2} F Q(P+Q)^{n}(I-(P+Q) \Delta)+\left[I-P P^{\dagger} G\right]$

$$
\sum_{n=0}^{\infty}(P+Q)^{n} F Q \Delta^{n+2}-P^{\dagger} G F Q \Delta+(I-F) \Delta+P^{\dagger} G F
$$

Case (i)a:

If $Q F=0$ and $F=I-P P^{\dagger} G$, then $P^{\dagger} G F=0,(P+Q)^{n}=P^{n} F$ Case (i) is $(P+Q)^{\dagger} G=\sum_{n=0}^{\infty}\left(P^{\dagger} G\right)^{n+2} F Q(P+Q)^{n}(I-(P+Q) \Delta)+\left(I-P P^{\dagger} G\right)$

$$
\begin{array}{r}
\sum_{n=0}^{\infty}(P+G)^{n} F Q \Delta^{n+2}-P^{\dagger} G F Q \Delta+(I-F) \Delta+P^{\dagger} G F \\
\sum_{n=0}^{\infty}\left(P^{\dagger} G\right)^{n+2} F Q(P+Q)^{n}=\sum_{n=0}^{\infty}\left(P^{\dagger} G\right)^{n}\left(P^{\dagger} G\right)^{2} F Q(P+Q)^{n}
\end{array}
$$




$$
=0
$$

$$
\begin{aligned}
\left(I-P P^{\dagger} G\right) \sum_{n=0}^{\infty}(P+Q)^{n} F Q \Delta^{n+2} & =F \sum_{n=0}^{\infty} P^{n} F Q \Delta^{n+2} \\
& =\sum_{n=0}^{\infty} P^{n} F^{2} Q \Delta^{n+2} \\
& =\sum_{n=0}^{\infty} P^{n} F Q \Delta^{n+2} \\
& =\sum_{n=0}^{\infty} P^{n}\left(I-P P^{\dagger} G\right) Q \Delta^{n+2}
\end{aligned}
$$

$-P^{\dagger} G F Q \Delta+(I-F) \Delta+P^{\dagger} G F=0+(I-F) \Delta+0$

$$
=P P^{\dagger} G \Delta
$$

Therefore $(P+Q)^{\dagger} G=\sum_{n=0}^{\infty} P^{n}\left(I-P P^{\dagger} G\right) G \Delta^{n+2}+P P^{\dagger} G \Delta$.

\section{Case (i).a.1:}

If $Q F=0, F=I-P P^{\dagger} G$ and $Q$ is quasi-nilpotent. Then by Corollary 2(3).

If $Q$ is quasi-nilpotent.

Then $(P+Q)^{\dagger} G=\sum_{n=0}^{\infty}\left(P^{\dagger} G\right)^{n+1}(-Q)^{n}=\left(I+P^{\dagger} Q\right)^{\dagger} G P^{\dagger} G$

Case (i).a.1:

If $Q F=0, F=I-P P^{\dagger} G$ and $Q$ is quasi-nilpotent. Then by Corollary 2(3). If $Q$ is quasi-nilpotent.

$$
\begin{aligned}
& \text { Then }(P+Q)^{\dagger} G=\sum_{n=0}^{\infty}\left(P^{\dagger} G\right)^{n+1}(-Q)^{n}=\left(I+P^{\dagger} Q\right)^{\dagger} G P^{\dagger} G \\
& (P+Q)^{\dagger} G=P^{\dagger}\left(I+P^{\dagger} Q\right)^{\dagger} G Q Q^{\dagger} G+\left(I-Q Q^{\dagger} G\right)\left(\sum_{n=0}^{\infty}(-Q)^{n}\left(P^{\dagger}\right)^{n+1}\right) G+ \\
& \left(\sum_{n=0}^{\infty}\left(Q^{\dagger}\right)^{n+1}\left(-P^{\dagger}\right)^{n}\right) G\left(I-P P^{\dagger} G\right) \\
& P^{\dagger}\left(I+P^{\dagger} Q\right)^{\dagger} G Q Q^{\dagger} G=0 \\
& \left(I-Q Q^{\dagger} G\right)\left(\sum_{n=0}^{\infty}(-Q)^{n}\left(P^{\dagger}\right)^{n+1}\right) G=0 \\
& \left(\sum_{n=0}^{\infty}(-Q)^{n}\left(P^{\dagger}\right)^{n+1}\right) G\left(I-P P^{\dagger} G\right)=\left(\sum_{n=0}^{\infty}(-Q)^{n}\left(P^{\dagger}\right)^{n+1}\right) G\left(I-P P^{\dagger} G\right) \\
& =\left(\sum_{n=0}^{\infty}(-Q)^{n}\left(P^{\dagger}\right)^{n+1}\right) G F \\
& =\left[\left(Q^{\dagger}\right)^{0+1}(-P)^{0}+\left(Q^{\dagger}\right)^{1+1}(-P)^{1}+\cdots\right] G F \\
& =Q^{\dagger}\left(I+Q^{\dagger} P\right)^{\dagger} G \cdot G F \\
& =Q^{\dagger}\left(I+Q^{\dagger} P\right)^{\dagger} F
\end{aligned}
$$


$P P^{\dagger} G \Delta=P P^{\dagger} G Q^{\dagger}\left(I+Q^{\dagger} P\right)^{\dagger} F$

Therefore Case (1.a) becomes,

$$
(P+Q)^{\dagger} G=\sum_{n=0}^{\infty} P^{n}\left(I-P P^{\dagger} G\right) Q \Delta^{n+2}+P P^{\dagger} G Q^{\dagger}(I+Q+P)^{\dagger} F .
$$

\section{Case (ii)a.2:}

If $Q F=F Q=0, F=I-P P^{\dagger} G$ and $Q$ is quasi-nilpotent, then Case (1.a.1) turns into

$$
\begin{aligned}
(P+Q)^{\dagger} G= & P^{\dagger}(I+P+Q)^{\dagger} G Q Q^{\dagger} G+\left(I-Q Q^{\dagger} G\right)\left[\sum_{n=0}^{\infty}(-Q)^{n}\left(P^{\dagger}\right)^{n+1}\right] \\
& G+\left[\sum_{n=0}^{\infty}\left(Q^{\dagger}\right)^{n+1}(-P)^{n}\right] G\left(I-P P^{\dagger} G\right) \\
= & 0+0\left[\sum_{n=0}^{\infty}\left(Q^{\dagger}\right)^{n+1}(-P)^{n}\right] G\left(I-P P^{\dagger} G\right) \\
= & Q^{\dagger}\left(I+Q^{\dagger} P\right)^{\dagger} G \cdot G\left(I-P P^{\dagger} G\right) \\
= & Q^{\dagger}\left(I+Q^{\dagger} P\right)^{\dagger} F
\end{aligned}
$$

Therefore $(P+Q)^{\dagger} G=Q^{\dagger}\left(I+Q^{\dagger} P\right)^{\dagger} F$.

Case (i)b:

If $Q F=0$ and $F=P P^{\dagger} G$, then $(P+Q)^{n} F=P^{n} F=F P^{n} F=F(P+Q)^{n} F$ So we have $\left(I-P P^{\dagger}\right) \sum_{n=0}^{\infty}(P+Q)^{n} F Q \Delta^{n+2}=0$

Since $-P^{\dagger} F Q \Delta+(I-F) \Delta+P^{\dagger} G F=\left(I-P^{\dagger} Q\right)(I-F) \Delta+P^{\dagger} G F$

$$
=\left(I+P^{\dagger} Q\right)-1 P^{\pi} \Delta+P^{\pi} F \text { and }
$$

$P^{\pi} \Delta=P^{\pi} \sum_{n=0}^{\infty}\left(Q^{\dagger}\right)^{n+1}(-P)^{n}$ by Corollary 2(3) and Equation (10), Case (1) becomes

$$
\begin{aligned}
(P+Q)^{\dagger} G= & \sum_{n=0}^{\infty}\left(P^{\dagger}\right)^{n+1} Q(P+Q)^{n}\left[I-(P+Q) \sum_{n=0}^{\infty}\left(Q^{\dagger}\right)^{n+1}(-P)^{n}\right] \\
& +\left(I+P^{\dagger} Q\right)^{-1} P^{\pi} \sum_{n=0}^{\infty}\left(Q^{\dagger}\right)^{n+1}(-P)^{n}+P^{\dagger}
\end{aligned}
$$

Case (i)b.1:

If $Q F=0, F=P P^{\dagger} G$ and $Q$ is quasi-nilpotent then Case (1.b) can be simplified as 


$$
\begin{aligned}
(P+Q)^{\dagger} G= & {\left[\sum_{n=0}^{\infty}\left(P^{\dagger}\right)^{n+2} Q(P+Q)^{n}\right] G\left[I-(P+Q) \sum_{n=0}^{\infty}\left(Q^{\dagger}\right)^{n+1}(-P)^{n}\right] G } \\
& +\left(I+P^{\dagger} Q\right)^{\dagger} G P^{\dagger}\left(\sum_{n=0}^{\infty}\left(Q^{\dagger}\right)^{n+1}(-P)^{n}\right) G+P^{\dagger} G \\
= & 0+\left(I+P^{\dagger} Q\right)^{\dagger} G\left(I-P P^{\dagger} G\right)\left[\sum_{n=0}^{\infty}\left(Q^{\dagger}\right)^{n+1}(-P)^{n}\right] G+P^{\dagger} G \\
= & 0+(I+0)^{\dagger} G(I-F)\left[\sum_{n=0}^{\infty}\left(Q^{\dagger}\right)^{n+1}(-P)^{n}\right] G+P^{\dagger} G \\
= & G(I-F) Q^{\dagger}\left(I+Q^{\dagger} P\right)^{\dagger} G G+P^{\dagger} G \\
(P+Q)^{\dagger} G= & G(I-F) Q^{\dagger}\left(I+Q^{\dagger} P\right)^{\dagger}+P^{\dagger} G .
\end{aligned}
$$

Case (i).b.2

If $Q F=F Q=0, F=P P^{\dagger} G$ and $Q$ is quasi-nilpotent then Case (1.b.1) becomes

$$
\begin{aligned}
(P+Q)^{\dagger} G & =G(I-F) Q^{\dagger}\left(I+Q^{\dagger} P\right)^{\dagger}+P^{\dagger} G \\
& =G(I-F) Q^{\dagger}\left(I+P^{\dagger} Q\right)+P^{\dagger} G \\
& =G(I-F) Q^{\dagger}(I+0)+P^{\dagger} G
\end{aligned}
$$

$(P+Q)^{\dagger} G=G(I-F) Q^{\dagger}+P^{\dagger} G$.

\section{Case (ii):}

If $Q F=(I-F) Q=0$, then $\Delta=P^{\dagger} G-P^{\dagger} G Q P^{\dagger} G,(I-F)(P+Q)^{n}=$ $(I-F) P^{n}$

$(P+Q)^{n} F=P^{n} F,(I-F)[I-(P+Q) 0]=(I-F)\left(I-P P^{\dagger} G\right)$ and $(I-F) \Delta=$ $(I-P) P^{\dagger} G$.

Theorem 2.13 reduces to

$$
\begin{gathered}
(P+Q)^{\dagger} G=\sum_{n=0}^{\infty} \Delta^{n+2} F Q(I-F)(P+Q)^{n}[I-(P+Q) \Delta]+[I-(P+Q) \Delta] \\
(P+Q)^{n} F Q(I-F) \Delta^{n+2}+(I-F) \Delta(I-\Delta F Q)+\Delta F \\
\sum_{n=0}^{\infty} \Delta^{n+2} F Q(I-F)(P+Q)^{n}[I-(P+Q) \Delta]=\sum_{n=0}^{\infty}\left(P^{\dagger}\right)^{n+2} Q P^{n}\left(I-P P^{\dagger} G\right) \\
{[I-(P+Q) \Delta](P+Q)^{n} F Q(I-F) \Delta^{n+2}=\left(I-P P^{\dagger} G\right) P^{n} Q\left(P^{\dagger}\right)^{n+2} .}
\end{gathered}
$$


Since $(I-F)(P+Q)^{n}=(I-F) P^{n}$

$$
\begin{aligned}
(I-F) \Delta(I-\Delta F Q)+\Delta F & =P^{\dagger} G+P^{\dagger} G-\left(P^{\dagger} Q P^{\dagger}\right) G \\
& =2 P^{\dagger} G-P^{\dagger} G Q P^{\dagger} G
\end{aligned}
$$

Therefore

$$
\begin{aligned}
(P+Q)^{\dagger} G= & \sum_{n=0}^{\infty}\left(P^{\dagger}\right)^{n+2} Q P^{n}\left(I-P P^{\dagger} G\right)+\left(I-P P^{\dagger} G\right) \sum_{n=0}^{\infty} P^{n} Q\left(P^{\dagger}\right)^{n+2} \\
& +2 P^{\dagger} G-P^{\dagger} G Q P^{\dagger} G .
\end{aligned}
$$

Case (ii)a:

If $Q F=(I-F) Q=P(I-F)=0$, then $Q P=Q P^{\dagger} G=0$. Then Case (ii) is

$$
\begin{aligned}
(P+Q)^{\dagger} G= & \sum_{n=0}^{\infty}\left(P^{\dagger}\right)^{n+2} Q P^{n}\left(I-P P^{\dagger} G\right)+\left(I-P P^{\dagger} G\right) \sum_{n=0}^{\infty} P^{n} Q\left(P^{\dagger}\right)^{n+2} \\
& +2 P^{\dagger} G-P^{\dagger} G Q P^{\dagger} Q \\
= & \left(P^{\dagger}\right)^{2} Q\left(I-P P^{\dagger} G\right)+\left(I-P P^{\dagger} G\right) Q\left(P^{\dagger}\right)^{2}+2 P+G-0 \\
= & \left(P^{\dagger}\right)^{2}\left(Q-Q P P^{\dagger} G\right)+\left(I-P P^{\dagger} G\right) 0+2 P^{\dagger} G \\
= & \left(P^{\dagger}\right)^{2} Q+2 P^{\dagger} G .
\end{aligned}
$$

\section{Case (ii)b:}

If $Q F=(I-F) Q=F P=0$, then $P Q=P^{\dagger} G Q=0$. Then Case (ii) turns

$$
\begin{aligned}
(P+Q)^{\dagger} G= & \sum_{n=0}^{\infty}\left(P^{\dagger}\right)^{n+2} Q P^{n}\left(I-P P^{\dagger} G\right)+\left(I-P P^{\dagger} G\right) \sum_{n=0}^{\infty} P^{n} Q\left(P^{\dagger}\right)^{n+2} \\
& +2 P^{\dagger} G-P^{\dagger} G Q P^{\dagger} G \\
= & \left(P^{\dagger}\right)^{2} a\left(I-P P^{\dagger} G\right)+\left(I-P P^{\dagger} G\right)(1) Q\left(P^{\dagger}\right)^{2} \\
& +2 P^{\dagger} G-0 \\
= & 0+\left(I-P P^{\dagger} G\right) 2 P^{\dagger^{2}}+2 P^{\dagger} G
\end{aligned}
$$$$
(P+Q)^{\dagger} G=Q\left(P^{\dagger}\right)^{2}+2 P^{\dagger} G
$$

\section{References}

[1]. A. Ben-Israel, T.N.E, Greville, Generalized Inverses: Theory and Applica- tions, Wiley-lnterscience, New York, 1974, second ed. Springer. New York, 2002.

[2]. N. Castro-Gonzalez, Additive perturbation results for the Drazin inverse, Linear Algebra Appl. 397 (2005) 279-297.

[3]. N. Castro-Gonzalez, JJ. Koliha, Perturbation of the Drazin inverse for closed linear operators, Integral Equations Operator Theory 36 (2000) 92- 106.

[4]. N. Castro-Gonzalez, J.J. Koliha, New additive results for the-Drazin in- verse, Proc. Roy. Soc. Edinburgh Sect. A 134 (2004) $1085-$ 1097. 
[5]. D.S. Cvetkovic-llic, D.S. Djordjevic, Y. Wei, Additive results for the gener- alized Drazin inverse in a Banach algebra, Linear Algebra Appl. 418 (2006) 53-61.

[6]. A. Dajic, J.J. Koliha, The weighted, g-Drazin inverse for operators, J. Aust. Math. Soc. 82 (2007) 163-181.

[7]. DS. Djordjevic, Y. Wei. Additive results for the generalized Drazin inverse, J. Aust. Math. Soc. 73 (2002) 115-125.

[8]. D.S. Djordjevic, P.S. Stanimirovic,On the generalized Drazin inverse and generalized resolvent, Czechoslovak Math. J. 51 (2001) 617-634.

[9]. M.P. Drazin, Pseudoinverse in associative rings and semigroups, Amer. Math. Monthly 65 (1958) 506-514

[10]. R.E. Hartwig, G. Wang, Y. Wei, Some additive results on Drazin inverse, Linear Algebra Appl. 322 (2001) 207-217.

[11]. J.J. Koliha, A generalized Drazin inverse, Glasg. Math, J. 38 (1996) 367- 381.

[12]. A.R. Meenakshi, Generalized inverses of matrices in Minkowski space, In Proc. Nat. Seminar Alg. Appln.Annamalai University, Annamalai Nagar (2000), 1-14.

[13]. M. Renardy, Singular value decomposition in Minkowski space, Linear Algebra Appl. 236 (1996), 53-58. 